\title{
IONOSPHERIC EFFECTS IN SAR INTERFEROMETRY: AN ANALYSIS AND COMPARISON OF METHODS FOR THEIR ESTIMATION
}

\author{
Ramon Brcic ${ }^{1}$, Alessandro Parizzi ${ }^{1}$, Michael Eineder ${ }^{1}$, Richard Bamler ${ }^{1}$ and Franz Meyer ${ }^{2}$ \\ ${ }^{1}$ Remote Sensing Technology Institute, German Aerospace Center (DLR), 82234 Wessling, Germany \\ Email: \{Ramon.Brcic, Alessandro.Parizzi, Michael.Eineder, Richard.Bamler\}@dlr.de \\ ${ }^{2}$ Geophysical Institute, University of Alaska, Fairbanks, AK, 99775, USA, Email: fmeyer@gi.alaska.edu
}

\begin{abstract}
For spaceborne SAR (Synthetic Aperture Radar) systems, the dispersive effects of the ionosphere on the propagation of the SAR signal can be a significant source of phase error. While at $\mathrm{X}$-band frequencies the effects are small, current and future P-, L- and C-band systems would benefit from ionospheric compensation to avoid errors in topographic retrieval. In this paper the focus is on the effects of the ionosphere on repeat-pass SAR interferometry from P- through X-bands and methods for their estimation which are demonstrated on L-band ALOS-PALSAR acquisitions ${ }^{1}$.
\end{abstract}

Index Terms - wideband interferometry, splitspectrum, delta-k, ionosphere

\section{INTRODUCTION - IONOSPHERIC EFFECTS IN SAR INTERFEROMETRY}

The ionosphere is a region of the earth's atmosphere extending from an altitude of about $50-1000 \mathrm{~km}$ where solar radiation ionizes atmospheric gases. For the purposes of SAR, it is sufficient to model the ionosphere as a thin layer at an effective ionospheric height of $h_{S P}=400 \mathrm{~km}$. During its 2-way passage through the dispersive ionosphere, a SAR signal experiences a group delay and phase advance which are inversely proportional to frequency squared

$$
-\tau_{\text {phase }}=\tau_{\text {group }}=2 K_{0} S T E C / f^{2}[\mathrm{~m}]
$$

where $K_{0}=40.28 \mathrm{~m}^{3} \mathrm{~s}^{-2}$ and STEC is the Slant range TEC (Total Electron Content). TEC refers to the zenith value, the density of free electrons contained in a cylinder with unit area passing through the atmosphere in the direction of nadir. STEC is proportional to TEC through one over the cosine of the zenith angle. For interferometry, only the phase delay is of interest. The corresponding influence on a repeat-pass interferogram is obtained by replacing STEC in

\footnotetext{
${ }^{1}$ ALOS-PALSAR data provided thanks to Americas ALOS Data Node (AADN) and JAXA. This work was supported by the European Space Agency, contract 21318/07/NL/HE.
}

Eqn (1) with $\triangle$ STEC, the difference in the $\triangle$ STEC values between the master and slave acquisitions, giving an interferometric phase delay at the carrier frequency $f_{\mathrm{C}}$ of

$$
\phi_{0}^{\text {iono }}=-\frac{2 K_{0} \Delta S T E C}{c f_{C}} \text { [cycles]. }
$$

Spatially, the ionosphere is usually considered homogeneous over the $\sim 100 \mathrm{~km}$ scale of an interferogram, possibly with azimuth variations at very high or low latitudes. Temporally, the sun-synchronous nature of the SAR systems orbits' means that master and slave acquisitions are imaged at the same mean local solar time. This removes much of the daily variability in the ionosphere leaving effects over larger time scales due to the seasons and the solar cycle.

In repeat-pass interferometry the dominant effect of the ionosphere is a phase screen in the interferogram that leads to topography retrieval errors. Given a constant $\triangle \mathrm{TEC}$ over an interferogram, the increase in signal path length through the ionosphere with slant range causes a range gradient in $\triangle \mathrm{STEC}$ and hence in the interferometric phase. Due to the low spatial variability of the ionosphere over the dimensions of a SAR scene this is usually the primary component of the ionospheric phase screen. Smaller secondary effects caused by spatial inhomogeneities in $\triangle \mathrm{TEC}$ on a scale smaller than the SAR scene dimensions act to further modulate this effect.

The focus here is estimation and correction of the phase screen to within a constant offset over the scene. For more details on the spatial and temporal behaviour of the ionosphere and the effects induced in repeat-pass interferometry and SAR in general such as azimuth shifts, defocus and Faraday rotation, see [1][3]-[7].

Table 1 lists the basic parameters of some current (ALOSPALSAR, TerraSAR-X) and future (BIOMASS, DESDynI/TerraSAR-L, Sentinel-1) P-, L-, C- and X-band spaceborne SAR systems and summarizes the effects of the ionosphere on the interferometric phase. From Table 1, a constant $\triangle$ TEC leads to a non-negligible phase gradient in the interferogram over range at $\mathrm{P}-$, L- and C-band. Furthermore, at P- and L-band a spatial inhomogeneity of only one TECU (one TEC Unit is equivalent to a TEC of 


\begin{tabular}{|c|c|c|c|c|c|c|c|}
\hline \multirow{6}{*}{$\begin{array}{c}\text { Band } \\
\text { Carrier frequency } \\
\text { Agency, Mission } \\
\text { Range bandwidth }[\mathrm{MHz}] \\
\text { Carrier frequency to bandwidth } \\
\text { ratio } \\
\text { Orbital height }[\mathrm{km}]\end{array}$} & \multirow{4}{*}{$\begin{array}{c}\mathrm{P} \\
435 \mathrm{MHz} \\
\text { ESA } \\
\text { BIOMASS } \\
6\end{array}$} & \multirow{2}{*}{\multicolumn{2}{|c|}{$\begin{array}{c}\mathrm{L} \\
1.27 \mathrm{GHz}\end{array}$}} & \multirow{6}{*}{$\begin{array}{c}\mathrm{C} \\
5.405 \mathrm{GHz} \\
\text { ESA Sentinel-1 } \\
100 \\
54 \\
693\end{array}$} & \multirow{3}{*}{\multicolumn{3}{|c|}{$\begin{array}{c}\text { X } \\
9.65 \mathrm{GHz} \\
\text { DLR TerraSAR-X }\end{array}$}} \\
\hline & & & & & & & \\
\hline & & \multirow{2}{*}{$\begin{array}{c}\text { NASA/DLR } \\
\text { DESDynl/TerraSAR-L } \\
80\end{array}$} & $\begin{array}{c}\text { JAXA } \\
\text { ALOS-PALSAR }\end{array}$ & & & & \\
\hline & & & 14 & & 100 & 150 & 300 \\
\hline & 73 & 16 & 45 & & 97 & 64 & 32 \\
\hline & 641 & 600 & 692 & & \multicolumn{3}{|c|}{514} \\
\hline $\begin{array}{c}\text { Interferometric phase advance } \\
\text { [cycles/TECU] }\end{array}$ & 7.5 & \multicolumn{2}{|l|}{2.6} & 0.61 & \multicolumn{3}{|c|}{0.34} \\
\hline Swath width $[\mathrm{km}]$ & 100 & \multicolumn{2}{|l|}{50} & 250 & \multicolumn{3}{|c|}{30} \\
\hline $\begin{array}{l}\text { Interferometric range phase } \\
\text { gradient [cycles/TECU] }\end{array}$ & 0.89 & \multicolumn{2}{|l|}{0.15} & 0.18 & \multicolumn{3}{|c|}{0.012} \\
\hline
\end{tabular}

Table 1: Parameters of the reference SAR systems. Ionosphere induced interferometric phase advance per (zenith) $\Delta$ TEC at $35^{\circ}$ incidence angle. Total change in interferometric phase over range per (zenith) $\triangle$ TEC assuming constant (zenith) $\Delta T E C$ over the scene for the specified swath width at $35^{\circ}$ incidence angle.

$10^{16}$ ) leads to ionospheric induced phase advances of several cycles, making correction vital.

\section{ESTIMATION OF IONOSPHERE INDUCED EFFECTS IN SAR INTERFEROMETRY}

From Eqn (2), the problems of ionospheric phase screen estimation and $\triangle$ STEC estimation are equivalent. Given the ionospheric phase screen for every pixel over the scene, the interferometric phase can be compensated for. The difficulty lies in separating the influence of the ionosphere from that of the topography and atmosphere. The three methods described below will be explored to achieve this.

\subsection{Split-spectrum method}

The interferometric phase at the carrier frequency is comprised of a non-dispersive topographic component (reference to the atmosphere is dropped since both the topographic and atmospheric phase behave equivalently w.r.t. frequency) due to the differential range between the master and slave and a dispersive ionospheric component due to the differential phase advance,

$$
\phi_{0}=\underbrace{2 f_{C} \Delta R / c}_{\phi_{0}^{\text {topo }}} \underbrace{-2 K_{0} \Delta S T E C /\left(c f_{C}\right)}_{\phi_{0}^{\text {iono }}} .
$$

The different behaviour of these two components w.r.t. frequency, namely the inverse frequency dependence of ionospheric phase delay versus the frequency proportional dependence of topographic phase delay, is exploited by the split-spectrum or delta-k method which employs subband processing in range to separate them [1]-[6]. From the theoretical performance of the split-spectrum method presented in [5], the carrier frequency to bandwidth ratio listed in Table 1 is a useful parameter for gauging performance changes between the various systems.

Table 2 compares the standard deviation of the $\triangle$ STEC and ionospheric phase estimates between the various systems in
Table 1 based on averaging over a constant area of $1 \mathrm{x} 1 \mathrm{~km}^{2}$ in slant range $x$ azimuth and a coherence of 0.7 . Note how the number of resolution cells contained within this $1 \mathrm{~km}^{2}$ patch over which averaging is carried out increases with range bandwidth - this allows good performance even at high carrier frequencies where the range bandwidth is greater. A comparison with the expected range phase gradient from Table 1 shows that conditions are the best for the BIOMASS and DESDynI/TerraSAR-L missions, are good at C-band for Sentinel-1 in interferometric wide-swath mode and are favorable even at X-band for TerraSAR-X given $300 \mathrm{MHz}$ acquisitions. For repeat-pass interferometry the effects of temporal decorrelation are expected to be less at lower frequencies which further favors the use of this technique for lower frequencies. These calculations assume that other dispersive error sources in the instrument can be neglected.

\subsubsection{Sensitivity to subband center frequency error}

The weights with which the subband phases are multiplied by before taking their difference are very close to one another. This makes the technique sensitive to errors in the subband center frequencies. For example, incorrect deweighting of the subband range spectra leading to a nonuniform distribution of energy effectively shift the subband range centre frequencies away from their nominal positions. When deviations do arise in practice, it is reasonable to assume that they are due to a non-uniform spectral weighting which is symmetric about the carrier frequency leading in turn to deviations in the subband center frequencies symmetric about $f_{\mathrm{C}}$. Assuming an increase (decrease) in the lower (upper) subband center frequencies of $\delta$, the carrier frequency will remain unaffected while the induced error in $f_{\Delta}$, the distance between the center frequencies, is $2 \delta$. The error in the split-spectrum estimate of ionospheric phase at the carrier frequency is then approximately 


\begin{tabular}{|c|ccccc|}
\hline Mission, Sensor & $\begin{array}{c}\text { Azimuth Resolution } \\
{[\mathrm{m}]}\end{array}$ & $\begin{array}{c}\text { Range Bandwidth } \\
{[\mathrm{MHz}]}\end{array}$ & $\begin{array}{c}\text { Resolution Cells } \\
\text { Averaged }\end{array}$ & $\begin{array}{c}\sigma(\Delta S T E C) \\
{[\text { TECU] }]}\end{array}$ & $\begin{array}{c}\sigma\left(\phi_{0}^{\text {iono }}\right) \\
\text { [cycles] }\end{array}$ \\
\hline BIOMASS & 12.5 & 6 & $3.2 \mathrm{k}$ & 0.044 & 0.27 \\
\hline ALOS-PALSAR & 4.5 & 14 & $21 \mathrm{k}$ & 0.063 & 0.13 \\
\hline DESDynl / TerraSAR-L & 4.5 & 28 & $42 \mathrm{k}$ & 0.022 & 0.047 \\
\hline Sentinel-1 & 10 & 80 & $53 \mathrm{k}$ & 0.0069 & 0.015 \\
\hline TerraSAR-X & 3.3 & 100 & $110 \mathrm{k}$ & 0.069 & 0.034 \\
\hline
\end{tabular}

Table 2: Theoretical standard deviation of the split-spectrum estimates for $\triangle$ STEC and the ionosphere induced interferometric phase for the various SAR systems from Table 1 assuming averaging over a constant area of $1 \mathrm{~km}$ in slant range $\mathrm{x} 1 \mathrm{~km}$ in azimuth with a coherence of 0.7 .

$$
-\frac{\delta}{f_{\Delta}}\left(\phi_{0}^{\text {iono }}-\phi_{0}^{\text {topo }}\right) .
$$

Frequency errors then induce a coupling between the ionospheric and topographic phase estimates with the proportion by which the ionospheric phase leaks into the topographic phase and vice versa being $\delta / f_{\Delta}$. Since the subband centre frequencies are usually known very accurately, this factor should normally be small. If this is not so, the coupling becomes significant for ionospheric (topographic) phase estimation when the change in topographic (ionospheric) phase over a scene is not small compared to the change in ionospheric (topographic) phase.

\subsection{Range group-phase delay difference method}

The difference in sign between the range group and phase delays due to the ionosphere in Eqn (1) can be exploited to estimate $\triangle \mathrm{STEC}[1][5]$. By subtracting the range phase delay, obtained from the unwrapped interferometric phase, from the range group delays as obtained from coregistration and after conversion to phase cycles, the common topographic component disappears, leaving only the ionosphere. This method does not require subband processing as in the split-spectrum method but does require highly accurate coregistration. This can be achieved through coherent cross-correlation between the master and slave over sufficiently large patches. Accurate estimation of the range phase delay by unwrapping the interferogram also requires a suitable amount of interferometric multilooking. After differencing the range group and phase delays, further smoothing can be applied to reduce the variance. Given that the number of resolution cells, $N$, used to obtain the final estimates of range group and phase delay are the same, the performance of the ionospheric phase estimate is dominated by that of the group delay estimate. If coherent (incoherent) cross-correlation is used, the standard deviation of the range group-phase delay difference method is reduced by a factor $3 / \sqrt{ } 2(3 / 2)$ compared to that of the split-spectrum method.

Aside from better statistical performance, this approach also has the advantage that unwrapping is only performed on the fullband phase whereas for split-spectrum a difference between the unwrapped subband phases is taken. The reduced bandwidth of the subbands combined with the two unwrapping operations may make the split-spectrum method more prone to phase unwrapping errors.

In terms of computational performance, this approach avoids the subband filtering and InSAR processing required by the split-spectrum method. Furthermore, if, as was the case here, coregistration was carried out using crosscorrelation, then the obtained range group delays can be reused for ionospheric phase estimation.

\subsection{Subband cross-correlation}

Both aforementioned estimators recover only the relative $\triangle$ STEC between any two points and are ambiguous w.r.t. a constant value over the scene. Estimation of the absolute $\triangle \mathrm{STEC}$ is possible in theory by performing highly accurate cross-correlation between suitably sized patches of the master and slave over at least 2 range subbands. The frequency proportional topographic component can then be separated from the inverse frequency proportional ionospheric component in a similar fashion as with the splitspectrum method. A theoretical analysis has shown that the standard deviation relative to that of the split-spectrum estimator is substantially increased by a factor of $\sqrt{3} f_{\mathrm{C}} / B$ $\left(\sqrt{ } 6 f_{\mathrm{C}} / B\right)$ for coherent (incoherent) cross-correlation. The increase in smoothing required to neutralize this makes recovery of the absolute $\triangle$ STEC via subband crosscorrelation difficult. Nevertheless, depending on the size of the correlation patch and the carrier frequency to bandwidth ratio, recovery is theoretically possible. Conditions are most favorable for the DESDynI/TerraSAR-L mission where given a coherence of 0.7 , estimation of $\triangle$ STEC with a standard deviation on the order of 0.1 TECU should be possible after averaging over $10^{5}$ resolution cells. In comparison, for the ALOS-PALSAR $14 \mathrm{MHz}$ acquisitions 

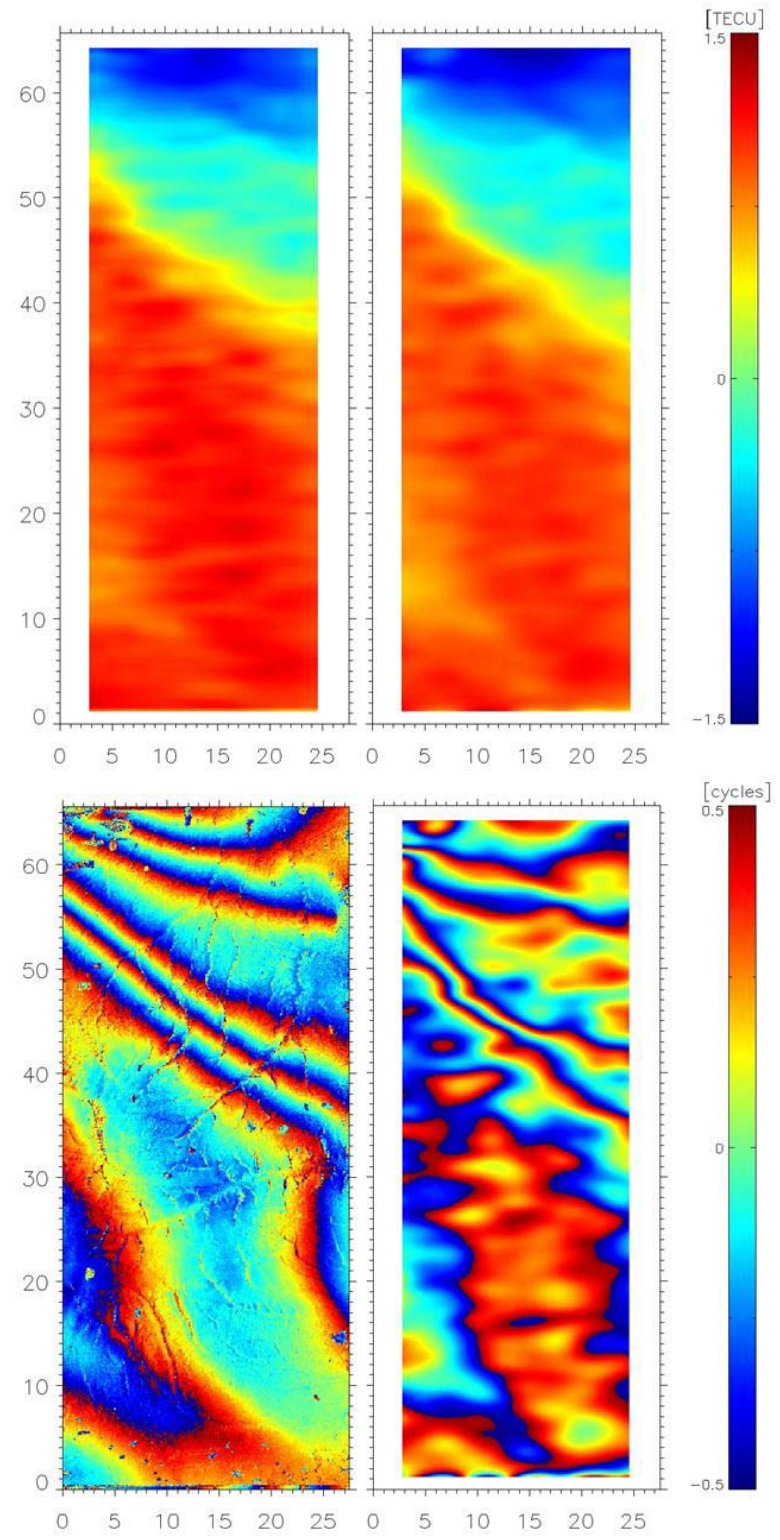

Fig. 1: Top: $\triangle$ STEC estimates from the range group-phase delay difference (left) and split-spectrum (right) methods. Bottom: DEM compensated phase (left) and rewrapped ionospheric phase estimate from the split-spectrum method (right). Ground range is on the x-axis and azimuth on the yaxis, both in $\mathrm{km}$.

used here, averaging over at least $10^{8}$ resolution cells would be required - rendering recovery impossible.

\section{EXPERIMENTS}

Figure 1 shows the estimated $\triangle$ STEC using the splitspectrum and range group-phase delay difference methods for two $14 \mathrm{MHz}$ bandwidth ALOS-PALSAR interferometric acquisitions taken over Alaska which are known to be dominated by a strong ionospheric component. The resolution in the ionosphere is roughly $2 \times 2 \mathrm{~km}$ for the range group-phase delay difference method and $1 \mathrm{x} 1 \mathrm{~km}$ for the split-spectrum method. Based on the average coherence of 0.5 , the theoretical standard deviation of the split-spectrum $\triangle \mathrm{STEC}$ estimate was $\sim 0.04 \mathrm{TECU}$ or $\sim 0.09$ cycles. Also shown is the DEM compensated phase and the rewrapped split-spectrum ionospheric phase estimate. The DEM compensated phase is essentially the ionospheric phase whose fringes match well with those estimated using the split-spectrum technique. Experiments with other acquisitions also showed similar good agreement between the methods for coherences $>0.5$. For coherences $<0.2$, phase unwrapping errors dominated leading to incorrect estimates and large differences between the methods.

\section{CONCLUSION}

The interferometric phase screens caused by the ionosphere at $\mathrm{P}$ and L-Band make compensation necessary for SAR systems at these frequencies. Theory and experimental results at L-band show that at least two interferometric methods - the split-spectrum and range group-phase delay difference techniques - can be used to estimate $\triangle$ STEC or equivalently the ionospheric phase screen, to within a constant offset. Recovery of this offset, which may be of interest in ionospheric research but is not relevant for interferometry, is theoretically possible for DESDynI/TerraSAR-L but not for the ALOS-PALSAR acquisitions used here.

\section{REFERENCES}

[1] F. Meyer, R. Bamler, N. Jakowski and T. Fritz, "The potential of low-frequency SAR systems for mapping ionospheric TEC distributions," IEEE Geoscience and Remote Sensing Letters, vol. 3, no. 4, pp. 560-564, October 2006.

[2] R. Bamler and M. Eineder. "Accuracy of differential shift estimation by correlation and split-bandwidth interferometry for wideband and delta-k SAR systems," IEEE Geoscience and Remote Sensing Letters, vol. 2, no. 2, pp. 151-155, April 2005.

[3] R. Brcic, M. Eineder and R. Bamler, "Interferometric absolute phase determination with TerraSAR-X wideband SAR data,", Proceedings of RadarCon 2009, Pasadena USA, May 2009.

[4] T. Freeman et al., "DESDynI, A NASA Mission for Ecosystems, Solid Earth and Cryosphere Science," Proceedings of PolInSAR 2009, Frascati Italy, January 2009.

[5] R. Brcic, A. Parizzi, M. Eineder, R. Bamler and F. Meyer, "Estimation and Compensation of Ionospheric Delay for SAR Interferometry," Proceedings of IGARSS 2010, Hawaii USA, July 2010.

[6] P. Rosen, S. Hensley, F. Meyer and T. Ainsworth, "Further developments in ionospheric mitigation of repeat-pass InSAR data," Proceedings of IGARSS 2010, Hawaii USA, July 2010.

[7] F. Meyer, "Performance requirements for correction of ionospheric signals in L-band SAR data," Proceedings of EUSAR 2010, Aachen Germany, June 2010. 